\title{
Efficiency of a solid-phase chemiluminescence immunoassay for detection of antinuclear and cytoplasmic autoantibodies compared with gold standard immunoprecipitation
}

\author{
Carmen Gelpí • Elena Pérez • Cristina Roldan
}

Received: 20 February 2014/ Accepted: 20 June 2014/Published online: 18 July 2014

(C) Springer-Verlag Italia 2014

\begin{abstract}
Purpose The aim of this study was to compare the degree of agreement of a novel Zenit RA chemiluminescent immunoassay (CLIA) from A. Menarini Diagnostics (Florence, Italy) and the gold standard immunoprecipitation assay to screen for the presence of specific antiU1snRNP, anti-Sm, anti-Ro/SS-A, anti-La/SS-B, anti-Jo1 ( ${ }^{\text {his }}$ tRNA-Synthetase) and anti-Scl-70(Topo I) antibodies. Materials and methods We studied 114 sera, 98 from patients with well-defined autoimmune connective tissue diseases and 16 from blood donor volunteers. All samples were fully characterized using the new chemiluminescent immunoassay and immunoprecipitation. In addition, all the samples were analyzed by indirect immunofluorescence (IIF) and anti-Scl-70(Topo I) antibodies were analyzed by immunoblot (IB) assay. Discrepant samples were analyzed using a commercial dot blot technique (Recomline from Mikrogen). The simple Kappa coefficient was used to measure the level of agreement between the results of Zenit RA CLIA and the gold standard.

Results The Kappa agreement between Zenit RA CLIA and gold standard immunoprecipitation, as well as IB and IIFassays for the presence of anti-Scl-70(Topo I)(0.948) was excellent. The concordance between Zenit RA CLIA and gold standard immunoprecipitation for the presence of anti-U1snRNP (0.883), anti-Ro/SS-A (0.878), anti-Jo$1\left({ }^{\text {his }}\right.$ tRNA-Synthetase) $(0.791)$ and anti-Sm (0.786) was
\end{abstract}

C. Gelpí $(\bowtie) \cdot$ E. Pérez

Department of Immunology, Hospital de la Santa Creu i Sant Pau, Avgda. S. Antoni $\mathrm{M}^{\mathrm{a}}$ Claret 167, 08025 Barcelona, Spain e-mail: mgelpi@santpau.cat

C. Gelpí · C. Roldan

Research Institute IBSantPau, Hospital de la Santa Creu i Sant Pau, Avgda. S. Antoni M ${ }^{a}$ Claret 167, 08025 Barcelona, Spain good, and excellent when the cut-off was raised to $14 \mathrm{U} / \mathrm{ml}$ (arbitrary units/ml). Between Zenit RA CLIA and gold standard immunoprecipitation for the presence of anti-La/ SS-B, the Kappa agreement had a value of 0.689 , but this improved to 0.775 when the cut-off was raised to $14 \mathrm{U} / \mathrm{ml}$. Precision was good based on the evaluation of replicate samples. Inter-assay coefficient variation was lower than $3.4 \%(\mathrm{CV}$ in \%) in all the kits and $<1.2 \%(\mathrm{CV}$ in \%) for intra-assay measurements.

Conclusion Our findings show that Zenit RA CLIA was specific and sensitive to detect anti-U1snRNP, anti-Sm, anti-Ro/SS-A, anti-La/SS-B, anti-Jo-1 ( ${ }^{\text {his }}$ tRNA-Synthetase) and anti-Scl70(Topo I) autoantibodies. This simple, fast and precise method can be a suitable option to analyze these autoantibody specificities.

Keywords Specificity - Sensitivity - Zenit RA chemiluminescent immunoassay - Immunoprecipitation . U1snRNP $\cdot$ Sm $\cdot$ Ro/SS-A $\cdot$ La/SS-B $\cdot$ Jo1 ( ${ }^{\text {his }}$ tRNAsynthetase) $\cdot$ Scl-70(Topo-I) $\cdot$ Autoantibodies $\cdot$ ENA

\section{Introduction}

Systemic autoimmune diseases are characterized by a systemic immunological response against widely distributed self-antigens. Representative autoimmune diseases included in this category are systemic lupus erythematosus (SLE), scleroderma or systemic sclerosis (SSc), polymyositis/dermatomyositis, Sjögren's syndrome (SS) and rheumatoid arthritis (RA). They are characterized by the production of antinuclear antibodies (ANA), at times more precisely referred to as anti-nuclear and cytoplasm antibodies (ANA-C) because of their localization in the cell nucleus and cytoplasm. The precise characterization of 
ANA is valuable for diagnosis. Anti-Sm, for example, are the most specific autoantibodies for SLE [1], anti-Jo-1 ( ${ }^{\text {his- }}$ tRNA-Synthetase) autoantibodies are specific for polymyositis and related anti-synthetase syndromes [1,2], and anti-Scl-70(Topo-I) autoantibodies [3] are specific for SSc [1].

Methods used to analyze ANA have developed greatly over the last decades. For many years, immunodiffusion, counterimmunoelectrophoresis, and indirect immunofluorescence (IIF) using rat tissue were standard use, but they have been replaced by other methods such as enzyme immunoassay (ELISA) and Western blot or immunoblot (IB). However, the reliability of the results obtained by ELISA and IB depends on the characteristics of the technique and the antigen source. Despite its low specificity, IIF using rat tissue and HeLa or HEp-2 cells is still used as an initial screening test because it is sensitive and provides information about the localization of the antigen in the cells [4]. However, other methods are needed to more precisely characterize ANA autoantibody specificities. The two methods that provide most benefit in characterizing autoantibody specificity are the RNA immunoprecipitation assay, first reported by Lerner and Steitz in 1979 [5], and the protein immunoprecipitation assay, reported by Matter et al. in 1982 [6]. Using the RNA immunoprecipitation assay, in 1982, Lerner, Steitz and Hardin identified a group of small nuclear and small cytoplasmic ribonucleoprotein $(\mathrm{sn} / \mathrm{scRNP})$ molecules as the major target antigens for the ANA autoantibodies in sera from patients with systemic autoimmune diseases [7]. These sn/scRNP antigens included a group of antigens referred to as extractable nuclear antigens (ENA) [8]. These ENA are the target for antiU1snRNP (small nuclear ribonucleoprotein), anti-Sm, antiRo/SS-A, anti-La/SS-B and anti-Jo-1 autoantibodies. The immunoprecipitation assay is considered the gold standard technique to study the fine specificity of the anti-sn/scRNPs antibodies. The protein immunoprecipitation assay reported by Matter is the gold standard for the screening of autoantibodies against protein antigens not associated with sn/sc RNA. An example of anti-nuclear autoantibodies reacting with an antigenic protein not associated with RNA are the anti-Scl-70 autoantibodies [3, 9].

These two gold standard techniques have been extensively used in clinical research laboratories but not in routine clinical laboratories where results are needed in a short time. Menarini Diagnostics recently launched a chemiluminiscent immunoassay (CLIA) system for use on the dedicated ZENIT RA analyzer (Zenit RA) based on autoantigen-coated magnetic particles as solid phase and an antibody labeled with a dimethyl acridinium ester as detection marker.

The aim of this paper was to compare the specificity and sensitivity of the Menarini Zenit RA CLIA kits with the
RNA immunoprecipitation technique to evaluate anti-sn/ scRNPs antibodies and also with the protein immunoprecitation technique to evaluate anti-Scl-70 antibodies. We also determined the intra-laboratory reproducibility of the Menarini Zenit RA CLIA kits and studied whether mixtures of antibodies with different specificities interfered with antibody binding and detection.

\section{Materials and methods}

Sera

One hundred and fourteen serum samples, 98 from patients with autoimmune connective tissue diseases (96 females and two males) and 16 from blood donor volunteers (15 females and 1 male), were selected from the sera bank of the Immunology Department of the Hospital de la Santa Creu i Sant Pau for the study. Patients mean age was 38 years (range 17-68 years). All the samples had been analyzed using gold standard immunoprecipitation assays. Of the 114 samples, 42 were from SLE patients, 35 from SS (including 11 patients that have SLE), four from PM, 1 from ASS, 11 from SSc, 3 from MCTD, 13 from connective tissue unrelated disease and 16 from healthy blood donor volunteers). Of the 42 patients with SLE, 25 had lupus nephritis, 6 patients (from whom we study eight samples) had neurological manifestations, three had APS and 11 had an associated secondary SS. Of the 11 patients with SSc, nine patients (from whom we studied 10 samples) had diffuse SSc and one had a limited cutaneous form of SSc. Of the nine patients with diffuse SSc, four had cutaneous involvement of the disease. Table 1 summarizes the clinical outcomes of the patients.

\section{Preparation of cell extracts}

HeLa cells were maintained at $37{ }^{\circ} \mathrm{C}, 5 \% \mathrm{CO}_{2}$ growing in $\log$ phase at $2 \times 10^{5}$ cells $/ \mathrm{ml}$, in RPMI- 1640 medium supplemented with $10 \%$ heat-inactivated foetal calf serum, $1 \%$ glutamine, $100 \mathrm{U} / \mathrm{ml}$ penicillin and $60 \mu \mathrm{g} / \mathrm{ml}$ streptomycin (GIBCO, Chagrin Falls, OH). Whole-cell extracts were prepared as described previously [10]).

\section{Analysis of immunoprecipitated RNPs}

To identify ANA capable of binding specific sn/scRNP, sera were tested for their ability to immunoprecipitate subsets of small RNAs from whole HeLa cells extracts. The standard assay method [5] and the silver-stained RNAs procedure were used [10]. To analyze the proteins immunoprecipitated by these antibodies, we used the standard method described by Matter [6], with modifications [11]. 
Table 1 Frequency of anti-Sm, anti-U1snRNP, anti-Ro/SS-A, anti-La/SS-B, anti-Jo-1 ( ${ }^{\text {his }}$ tRNA-Synthetase) and anti-Scl-70(Topoisomerase I) autoantibodies in patient sera

\begin{tabular}{|c|c|c|c|c|c|c|c|}
\hline & anti-U1snRNP & anti-Sm & anti-Ro/SS-A & anti-La/SS-B & anti-Jo-1 & anti-Scl-70 & Other anti-rRNP \\
\hline $\operatorname{SLE}(n=42)$ & 19 & 6 & 30 & 13 & 0 & 0 & 7 \\
\hline $\operatorname{SLE}+\operatorname{SS}(n=11)$ & 1 & 1 & 11 & 7 & 0 & 0 & 0 \\
\hline $\mathrm{SLE}+\mathrm{RD}(n=5)$ & 4 & 0 & 1 & 1 & 0 & 0 & 0 \\
\hline SLE + NS $(n=8)$ & 9 & 1 & 2 & 1 & 0 & 0 & 6 \\
\hline $\operatorname{SLE}+\operatorname{APS}(n=3)$ & 2 & 0 & 0 & 0 & 0 & 0 & $2(\mathrm{NS})$ \\
\hline $\operatorname{SS}(n=24)$ & 1 & 0 & 24 & 11 & 0 & 0 & 0 \\
\hline $\mathrm{SS}+\mathrm{RA}(n=3)$ & 0 & 0 & 3 & 2 & 0 & 0 & 0 \\
\hline $\mathrm{SS}+\operatorname{APS}(n=2)$ & 0 & 0 & 2 & 0 & 0 & 0 & 0 \\
\hline $\mathrm{SS}+\mathrm{PBC}(n=3)$ & 0 & 0 & 3 & 0 & 0 & 0 & 0 \\
\hline $\mathrm{PM}(n=4)$ & 0 & 0 & 3 & 0 & 4 & 0 & 0 \\
\hline $\mathrm{PM}+\mathrm{SS}(n=1)$ & 0 & 0 & 1 & 0 & 1 & 0 & 0 \\
\hline $\operatorname{ASS}(n=1)$ & 0 & 0 & 1 & 0 & 1 & 0 & 0 \\
\hline $\operatorname{SSc}(n=11)$ & 4 & 0 & 0 & 0 & 0 & 10 & 0 \\
\hline $\operatorname{MCTD}(n=3)$ & 3 & 0 & 0 & 0 & 0 & 0 & 0 \\
\hline Other $(n=13)$ & 6 & 0 & 7 & 0 & 0 & 0 & 0 \\
\hline NHS $(n=16)$ & 0 & 0 & 0 & 0 & 0 & 0 & 0 \\
\hline Prevalence & $27.8 \%$ & $6.7 \%$ & $56.7 \%$ & $23 \%$ & $4.8 \%$ & $9.6 \%$ & \\
\hline
\end{tabular}

Gold standard techniques were used to calculate the frequency of autoantibodies in our cohort of patient sera. The left column shows the diagnosis and number of the patient sera analyzed. The second column shows the number of patients with more than one associated autoimmune disease

$S L E$ systemic lupus erythematosus, $S S$ Sjögren's syndrome, $R D$ renal disease, $N S$ neurological syndrome, $A P S$ antiphospolipid syndrome, $R A$ rheumatoid arthritis, $P B C$ primary biliary cirrhosis, $P M$ polymyositis, $A S S$ antisynthetase syndrome, SSc systemic sclerosis, MCTD mixed connective tissue disease, $N H S$ normal human sera

\section{Indirect immunofluorescence}

Commercially available Hep-2 cells (Euroimmun) and HeLa cells grown on glass slides in our laboratory were used as described [11].

\section{Immunoblots}

IB were performed as described by Towbin, Staehelin \& Gordon [12], using Scl-70(Topo I) enriched extracts prepared as described [9].

\section{CLIA}

Antibodies were detected using the commercial CLIA methods from Menarini Diagnostics (Florence, Italy). The manufacturer of Zenit RA CLIA received the blind coded sera selected for this study from the Immunology Department at Hospital de la Santa Creu i Sant Pau and reported the results in units per milliliter.

Zenit RA CLIA consists of six kits, each containing a specific antigen attached to solid phase microspheres. The six specific antigens are native $\mathrm{Sm}$, recombinant $70, \mathrm{~A}$ and $\mathrm{C} \mathrm{U} 1$ RNP antigenic proteins, recombinant Ro60 and Ro52 proteins of the Ro/SS-A antigen, recombinant $48 \mathrm{kDa} \mathrm{La} / \mathrm{SS}$ $\mathrm{B}$ antigenic protein, recombinant $58 \mathrm{kDa}$ Jo-1 tRNA antigenic protein, and recombinant $72 \mathrm{kDa}$ protein of the Scl70antigen. The concentrations are expressed in arbitrary units $/ \mathrm{ml}$.

Discrepant samples were analysed by the manufacturer using a commercial dot blot kit Recomline (RM) from Mikrogen, (Neuss, Germany).

\section{Data analysis}

For each kit, operating characteristics (specificity and sensitivity for each ANA) were determined using the pooled data of all test samples. The nonparametric Kaplan procedure was used to estimate the degree of concordance between Zenit RA CLIA and gold standard immunoprecipitation [13].

A logistic regression model (receiver operating characteristic curve, ROC) with an AUC (area under curve) as discrimination coefficient (acceptable discrimination, AUC > 0.7) was constructed to evaluate the discriminant capacity of each parameter and its possible use as a diagnostic test $[13,14]$. All statistical analyses were performed 
using 19 SPSS statistical software (International Business Machines (IBM), New York, USA).

Intra-laboratory variability was assessed with coefficients of variation (CV). $\mathrm{CV}$ was calculated from the optical density (OD) (or units/ml) at the recommended serum dilution, using the replicated results with the duplicate test samples.

\section{Results}

Of the 114 samples analyzed, 89 were positive for at least one of the anti-U1snRNP, anti-Sm, anti-Ro/SS-A, anti-La/ SS-B, anti-Scl-70 and anti-Jo-1 ( ${ }^{\text {his }}$ tRNA-Synthetase) antibodies, and 25 were negative for all of them.

Table 1 shows the frequency of serological positivity using the gold standard technique in the sample of patient sera studied.

Figure 1 shows the comparison of frequencies for antiSm, anti-U1snRNP, anti-Ro/SS-A, anti-La/SS-B, anti-Jo1and anti-Scl-70 autoantibodies using Zenit RA CLIA and the gold standard assays.

Table 2 shows the Kappa agreement between Zenit RA CLIA and the gold standard immunoprecipitation. Using the manufacturer's recommended cut-off values we found an excellent concordance between Zenit RA CLIA and the presence of anti-Scl-70 (0.948), and a good concordance between Zenit RA CLIA and the presence of anti-U1snRNP (0.883), anti-Ro/SS-A (0.878), anti-Jo-1 (0.791) and anti-Sm (0.786), but only a moderate concordance with Zenit RA CLIA and the presence of antiLa/SS-B (0.689).

Figure 2 shows the discrimination capacity of all six kits. Specificity $\left(\mathrm{Sp}_{\mathrm{ROC}}\right)$ and sensitivity $\left(\mathrm{Sn}_{\mathrm{ROC}}\right)$ were calculated for each biomarker using ROC curves. Sm.

We found a good Kappa agreement (0.786) between Zenit RA CLIA and the presence of anti-Sm antibodies using the gold standard immunoprecipitation test. The comparative analysis of anti-Sm antibodies showed an area under the curve of 0.998 with a typical error of 0.003. Using ROC analysis with the manufacturer's recommended cut-off value of $10 \mathrm{U} / \mathrm{ml}$, the assay specificity was $98 \%$ and sensitivity of the Zenit RA CLIA test was $100 \%$. One sample from a patient with an overlap SS, PBC and APS gave a false positive probably as a result of matrix interference because it had a high OD value with all tests and was positive for all but one (anti-Ro/ SS-A) Zenit RA CLIA tests. When this sample was analyzed using the RM assay and IIF, the results were negative. The specificity for anti-Sm and all other markers was conditioned by the false positive of this sample; it was otherwise $100 \%$.

\section{U1 RNP}

We found a good Kappa agreement (0.883) between Zenit RA CLIA and the presence of anti-U1snRNP antibodies using the gold standard immunoprecipitation test. The ROC comparative analysis of the anti-U1snRNP antibodies showed an area under the curve of 0.989 with a typical error of 0.008. Using ROC analysis with a cut-off of $10 \mathrm{U} /$ $\mathrm{ml}$, specificity and sensitivity of the assay were 96.5 and $96.6 \%$, respectively. Four samples showed discrepancies with the gold standard. Besides the nonspecific sample already mentioned above, two samples from patients diagnosed of SLE were positive by Zenit RA CLIA and negative using the gold standard. The fourth discrepant sample, from SLE patients with renal and central nervous system (CNS) involvement and associated APS was positive using the gold standard and negative using Zenit RA CLIA and the RM technique. When this sample was tested by IIF using rat tissue and Hep-2 cells, the result was positive, showing two patterns: a speckled nuclear pattern that was compatible with anti-snRNP, and a nucleolus and cytoplasm pattern that was compatible with anti-ribosomal RNP antibodies. This serum had anti-ribosomal RNP in addition to the anti-U1snRNP antibodies.

\section{Ro/SS-A}

We found a good Kappa agreement (0.878) between Zenit RA CLIA and the presence of anti-Ro/SS-A antibodies using the gold standard. ROC analyses showed an area under the curve of 0.981 with a typical error of 0.015 . At the recommended cut-off of $10 \mathrm{U} / \mathrm{ml}$, specificity was $100 \%$ and sensitivity was $95 \%$. No false-positive results were found by Zenit RA CLIA. Three samples that were positive using the gold standard were not detected by Zenit RA CLIA. One of these samples, from a patient with SS and APS only recognized the hY5 scRNA particle from the Ro antigen and the other two samples, both from SLE patients, recognized the whole Ro molecule but had a low titre using the RNA immunoprecipitation gold standard assay.

\section{$\mathrm{La} / \mathrm{SS}-\mathrm{B}$}

Using the manufacturer's recommended cut-off, we found a moderate Kappa agreement (0.689) between Zenit RA CLIA and the presence of anti-La/SS-B (GS). Differences of anti-La/SS-B antibodies between Zenit RA CLIA and gold standard assays were due to two false positives (the already-mentioned nonspecific sample and a sample from a SLE patient with negative anti-SS-A/Ro antibodies by gold standard and CLIA tests) and five false negative Zenit RA CLIA results. Four of the five false negatives from patients 
Fig. 1 Prevalence of anti-Sm, anti-U1snRNP, anti-Ro/SS-A, anti-La/SS-B, anti-Jo-

1( ${ }^{\text {his }}$ tRNA-Synthetase) and antiScl70(Topo I) autoantibodies determined by CLIA or gold standard (GS) techniques

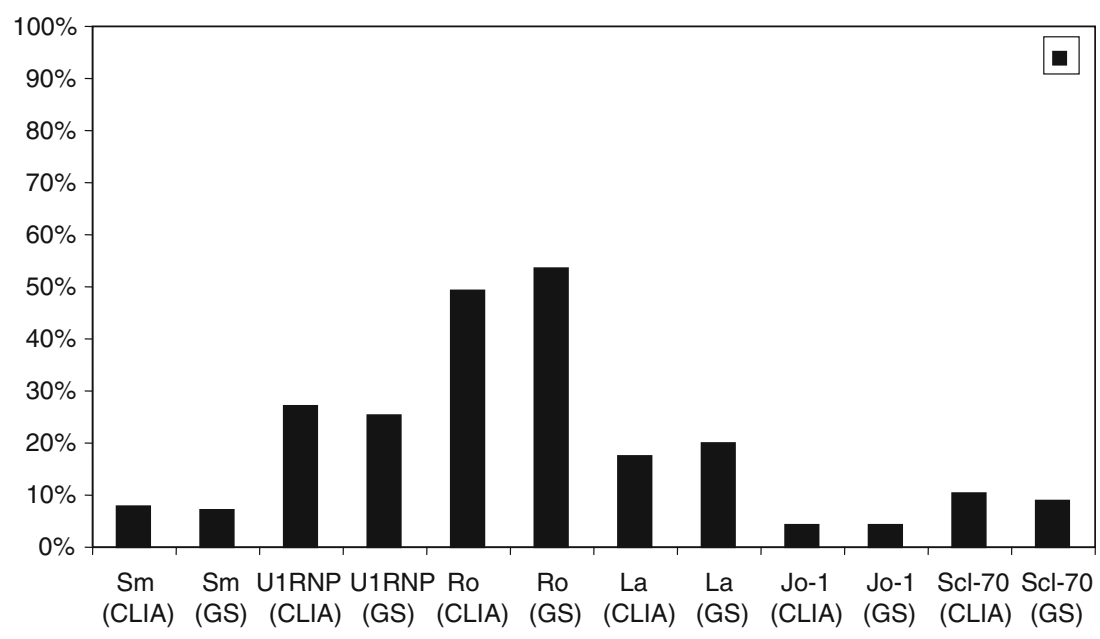

Table 2 Kappa agreement between Zenit RA CLIA results and the gold standard assays to detect anti-Sm, anti-U1snRNP, anti-Ro/SS-A, anti-La/SS-B, anti-Jo-1 ( ${ }^{\text {his }}$ tRNA-Synthetase) and anti-Scl-70(Topoisomerase I) autoantibodies at two different cut-offs

\begin{tabular}{lcll}
\hline Level & Sensitivity & Specificity & $k$ \\
Anti-Sm & & & \\
$10 \mathrm{U} / \mathrm{ml}$ & $100 \%$ & $98 \%$ & 0.786 \\
$14 \mathrm{U} / \mathrm{ml}$ & $100 \%$ & $99 \%$ & 0.936 \\
Anti-U1snRNP & & & \\
$10 \mathrm{U} / \mathrm{ml}$ & $96.6 \%$ & $96.5 \%$ & 0.883 \\
$14 \mathrm{U} / \mathrm{ml}$ & $86.2 \%$ & $97.6 \%$ & 0.910 \\
Anti-Ro/SS-A & & & \\
$10 \mathrm{U} / \mathrm{ml}$ & $95 \%$ & $100 \%$ & 0.878 \\
$14 \mathrm{U} / \mathrm{ml}$ & $90 \%$ & $100 \%$ & 0.947 \\
Anti-La/SS-B & & $97.8 \%$ & 0.689 \\
$10 \mathrm{U} / \mathrm{ml}$ & $75 \%$ & $98.9 \%$ & 0.775 \\
$14 \mathrm{U} / \mathrm{ml}$ & $70 \%$ & & 0.791 \\
Anti-Jo-1(histRNA-synthetase) & $98 \%$ & 0.791 \\
$10 \mathrm{U} / \mathrm{ml}$ & $80 \%$ & $99 \%$ & 0.948 \\
$14 \mathrm{U} / \mathrm{ml}$ & $80 \%$ & & 0.899 \\
Anti-Scl-70(Top-I) & & $98 \%$ & \\
$10 \mathrm{U} / \mathrm{ml}$ & $100 \%$ & & \\
$14 \mathrm{U} / \mathrm{ml}$ & $100 \%$ & & \\
\hline
\end{tabular}

diagnosed as SLE, one associated with a CNS involvement, one with SS and one with AFS had a low reaction in the RNA immunoprecipitation gold standard assay. The fifth sample was from a patient with Sicca syndrome. All five sera provided a speckled and nucleoli pattern using IIF with rat tissue and Hep-2 cells, compatible with anti-La/ SS-B antibodies.

The comparative ROC analysis of anti-La/SS-B antibodies showed an area under the curve of 0.929 with a typical error of 0.039 . At the recommended cut-off value of $10 \mathrm{U} / \mathrm{ml}$, specificity of the assay was $97.8 \%$ and sensitivity was $75 \%$. Using ROC analysis to improve the specificity of the assay to $98.9 \%$, the cut-off was $14 \mathrm{U} / \mathrm{ml}$. Kappa agreement with the gold standard increased to 0.775 (good agreement), although the sensitivity of the assay decreased from $75 \%$ to $70 \%$ (shown in Table 2).

Jo-1

We found a good Kappa agreement (0.791) between Zenit RA CLIA and the presence of anti-Jo-1 ( ${ }^{\text {his }}$ tRNA-synthetase) antibodies using the gold standard immunoprecipitation assay. The comparative ROC analysis of antiJo1 ( ${ }^{\text {his }}$ tRNA-Synthetase) antibodies showed an area under the curve of 0.930 with a typical error of 0.064 . Using ROC analysis, specificity of the assay was $98 \%$ and sensitivity was $80 \%$ for a cut-off value of $10 \mathrm{U} / \mathrm{ml}$. Increasing the cut-off to 14 , specificity rose to $99 \%$ and sensitivity remained at $80 \%$. Two differences between Zenit RA CLIA and the gold standard results were found. One difference was the already-mentioned nonspecific Zenit RA CLIA false positive result. The other difference was found in a sample from a patient with polymyositis, Grave's syndrome and associated diabetes mellitus. Gold standard immunoprecipitation assays showed this patient had antiJo-1 and anti-Ro/SS-A antibodies a Zenit RA CLIA gave anti-Jo-1 negative result. This sample was also negative by RM.

\section{Scl-70}

We found an excellent Kappa agreement (0.948) between Zenit RA CLIA and the presence of anti-Scl-70 antibodies using gold standard immunoprecipitation and immunoblot. Comparative analysis of anti-Scl-70 antibodies showed an area under the curve of 1 with a typical error of 0.000 . With the recommended cut-off value of $10 \mathrm{U} / \mathrm{ml}$, specificity of 

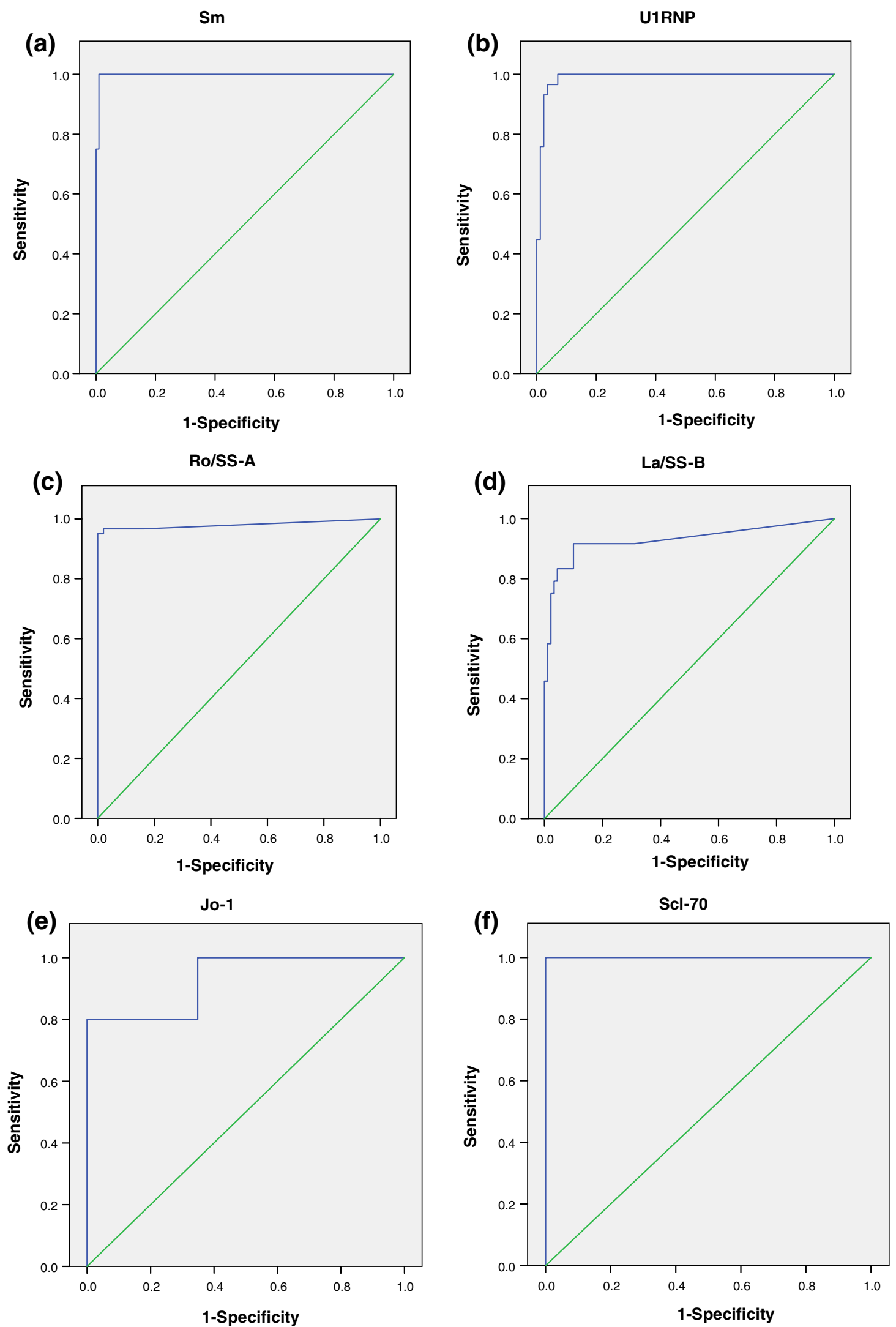

Fig. 2 ROC curve analyses of a anti-Sm, b anti-U1snRNP, $\mathbf{c}$ anti-Ro/SS-A, $\mathbf{d}$ anti-La/SS-B, e anti-Jo-1 ${ }^{\text {his }}$ tRNA-Synthetase) and $\mathbf{f}$ anti-Scl70(Topo I) autoantibodies 
the assay was $97 \%$ and sensitivity was $100 \%$. Using ROC analysis we found specificity of the assay increased to $98 \%$ with the same sensitivity of $100 \%$, using a cut-off value of $14 \mathrm{U} / \mathrm{ml}$. Table 2 shows the selection criteria and cut-off values with their corresponding sensitivity and specificity for the six tests.

We found that mixtures of antibodies of different specificities interfered with their detection by CLIA. In the patient cohort, 47 patients harbored one autoantibody specificity, 35 patients harbored two, 4 patients harbored three, and 1 patient harbored four. Two sera from a SLE patients with renal and CNS involvement and associated APS had anti-U1snRNP and anti-ribosomal RNP antibodies by immunoprecipitation assays, but they were negative or had a low level of anti-U1snRNP antibodies using Zenit RA CLIA. IIF was useful to confirm the presence of these two antibody specificities in these two sera. Two samples from a patient with polymyositis and Grave's syndrome and associated diabetes mellitus had anti-Ro/SS-A and anti-Jo-1 antibodies. Both antibody specificities were clearly detected by gold standard immunoprecipitation but not by Zenit RA CLIA. Zenit RA CLIA only detected the anti-Ro/SS-A antibodies. Only one serum from this patient was included in the study. No differences in the rate of agreement between the gold standard and Zenit RA CLIA were found related to other mixtures of antibody specificities present in the sera.

Intra-laboratory reproducibility and precision using the Zenit RA CLIA kits were good for all the assays. Interassay coefficient variation was lower than $3.4 \%$ (CV in $\%)$ in all the kits and $<1.2 \%$ (CV in \%) for intra-assay measurements.

\section{Discussion}

We found that Zenit RA CLIA had a good Kappa agreement with gold standard techniques to determine anti-Sm, anti-U1snRNP, anti-Ro/SS-A, anti-La/SS-B, anti-Jo-1 and anti-Scl-70 antibodies. However, we suggest the cut-off value recommended by the manufacturer should be increased to obtain an even higher coefficient of agreement.

Discrepancies between Zenit RA CLIA and immunoprecipitation gold standards were minimal and only influenced the degree of agreement or Kappa coefficient when sample size was small, as for example in anti-Sm and antiJo-1 antibodies. The discrepancy of the sample from a patient with polymyositis and associated Grave's and diabetes mellitus could be explained by the fact that Zenit RA CLIA and RM assays use recombinant proteins as the source of antigens while immunoprecipitation uses native proteins. The antigen target for anti-Jo-1 autoantibodies present in the patient sera may recognize other structural antigenic epitopes present in the native form of the protein but not in the recombinants. Having compared Zenit RA CLIA, IP, IB and IIF, and considering both specificity and sensitivity, we conclude that greater sensitivity does not guarantee a larger number of truly positive samples. ELISA and IB kits may provide positive reactions that are not seen with techniques such as IP or IIF assays. This positive results not detected by IP or IIF could be due to a higher sensitivity of the ELISA or IB kit or to unspecific binding of serum proteins to the solid phase of the kits. The higher specificity of some ELISA or IB kits is often due to low affinity autoantibodies detection. This increase in assay sensitivity would be at the cost of decreased disease specificity. Some commercially available ELISA or IB anti-La/SS-B and anti-Sm kits are examples of this detection of low affinity antibodies in patients without SS or SLE patient sera. Because anti-Sm antibodies have been included in the American College of Rheumatology diagnostic criteria of SLE, it is of particular concern that anti$\mathrm{Sm}$ autoantibodies have been reported in patients without SLE $[15,16]$. Anti-La/SS-B is rarely detected without antiRo because these two antigens share a common RNA termed hYRNA $[5,7,11]$. Anti-Sm antibodies are rarely found without anti-U1snRNP because both proteins associate with common snRNA species in the spliceosome [5, 7]. The detection of low-affinity antibodies that are not relevant in pathogenesis and do not show a relationship with disease does not provide any valuable information to the clinician.

The main limitation of our study is that the number of samples with anti-Sm autoantibodies, the number of patients with anti-Jo-1 autoantibodies and the number of patients with anti-Scl-70 autoantibodies was lower than the number of samples included in the study of anti-U1snRNP, anti-Ro/SS-A and anti-La/SS-B autoantibodies. A higher number of samples would have increased the Kappa agreement despite the two errors in the comparative study of anti-Jo-1 autoantibodies.

In conclusion, our results show that the Menarini Zenit RA CLIA method for detection of anti-Sm, anti-U1snRNP, anti-Ro/SS-A, anti-La/SS-B, anti-Jo-1 and anti-Scl-70 autoantibodies is specific and sensitive, and provides an easy, precise and useful test for the screening of these autoantibodies. However, we recommend using IIF as a first step algorithm that will provide a second assay to confirm diagnosis. In our experience Zenit RA CLIA and IIF or Immunoblot are simple confirmatory assays that provide sensitive and specific results in systemic autoimmune diseases.

Acknowledgments The authors would like to thank Dr Ignasi Gich for statistical assistance, Cristina Sanchez from Menarini Diagnostics 
(Barcelona) for technical assistance in the Zenit RA CLIA procedures and Carolyn Newey for help with the English. This study was supported by an unrestricted grant from Menarini, SA.

\section{Conflict of interest None.}

Human and animal rights The authors declare that human rights have been preserved and no animals have been used in the development of this study.

\section{References}

1. Tan EM, Chan EK, Sullivan KF, Rubin RL (1988) Antinuclear antibodies (ANAs): diagnostically specific immune markers and clues toward the understanding of systemic autoimmunity. Clin Immunol Immunopathol 47:121-141

2. Gelpi C, Kanterewicz E, Gratacos J, Targoff IN et al (1996) Coexistence of two antisynthetases in a patient with the antisynthetase síndrome. Arthritis Rheum 39:692-697

3. Douvas A (1988) Does Scl-70 modulate collagen production in systemic sclerosis? Lancet 27:475-477

4. Meroni PL, Schur PH (2010) ANA screening: an old test with new recommendations. Ann Rheum Dis 69:1420-1422

5. Lerner MR, Steitz JA (1979) Antibodies to small nuclear RNAs complexed with proteins are produced by patients with systemic lupus erythematosus. Proc Natl Acad Sci USA 76:5495-5499

6. Matter L, Schpfer K, Wilhelm JA et al (1982) Molecular characterization of ribonucleoprotein antigens bound by antinuclear antibodies: a diagnostic evaluation. Arthritis Rheum 25:1278

7. Hardin JA, Rahn DR, Shen C et al (1982) Antibodies from patients with connective tissue diseases bind specific subsets of cellular RNA-protein particles. J Clin Invest 70:141-147
8. Sharp GC, Irvin WS, Tan EM et al (1972) Mixed connective tissue disease - an apparently distinct rheumatic disease syndrome associated with a specific antibody to an extractable nuclear antigen (ENA). Am J Med 52:148-159

9. Juarez C, Vila JL, Gelpi C et al (1988) Characterization of the antigen reactive with anti-Scl-70 antibodies and its application in an enzyme-linked immunosorbent assay. Arthritis Rheum $31: 108-115$

10. Forman MS, Nakamura M, Mimori T, Gelpi C et al (1985) Detection of antibodies to small nuclear ribonucleoproteins and small cytoplasmic ribonucleoproteins using unlabeled cell extracts. Arthritis Rheum 28:1356-1361

11. Gelpi C, Alguero A, Martinez MA et al (1990) Identification of protein components reactive with anti-PM/Scl autoantibodies. Clin Exp Immunol 81:59-64

12. Towbin H, Staehelin T, Gordon J (1979) Electrophoretic transfer of proteins from polyacrylamide gels to nitrocellulose sheets: procedure and some applications. Biotechnology 24:145-149

13. Hanley JA, McNeil BJ (1982) The meaning and use of the area under a receiver operating characteristic (ROC) curve. Radiology 143:29

14. Greiner M, Sohr D, Göbel P (1995) A modified ROC analysis for the selection of cut-off values and the definition of intermediate results of serodiagnostic tests. J Immunol Methods 185:123-132

15. Tan EM, Cohen AS, Fries JF et al (1982) The 1982 revised criteria for the classification of systemic lupus erythematosus. Arthritis Rheum 25:1271-1277

16. Egner W (2000) The use of laboratory tests in the diagnosis of SLE. J Clin Pathol 53:424-432 